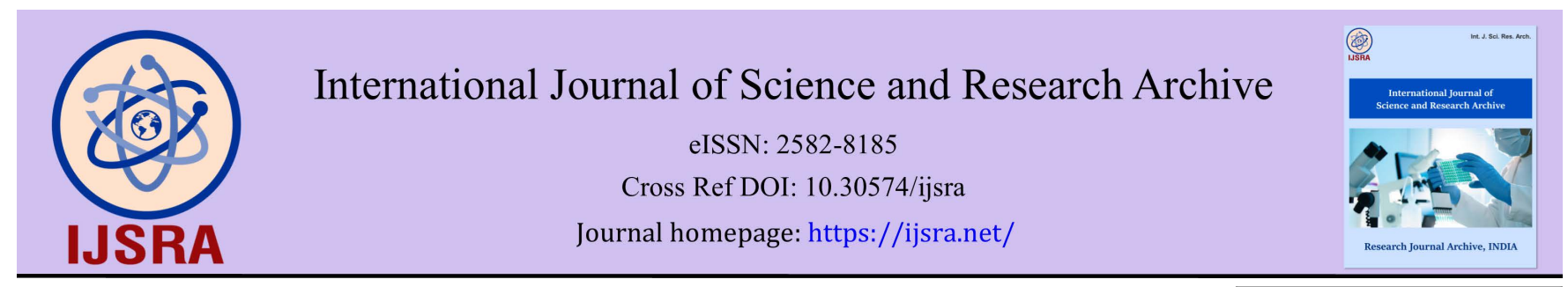

(REVIEW ARTiCLE)

\title{
Neurobiology for human suicide
}

\author{
Da-Yong Lu *, Hong-Ying Wu, Bin Xu and Ting-Ren Lu \\ School of Life Sciences, Shanghai University, Shanghai 200444, PRC. China.
}

International Journal of Science and Research Archive, 2021, 02(02), 120-125

Publication history: Received on 27 March 2021; revised on 05 May 2021; accepted on 08 May 2021

Article DOI: https://doi.org/10.30574/ijsra.2021.2.2.0056

\begin{abstract}
In the future, suicide risk prediction and management should be transformed from psychiatrist's judgment (mental illness-like symptoms) into modern technique-guided diagnostics and target drug therapeutics in larger clinical settings. To embrace modern scientific and technological advances, a number of unique diagnostic and therapeutic approaches must be established. This Article provides some important ideas and mechanisms proposed by translational programs.
\end{abstract}

Keywords: Suicide; Mood disorder; Neuropsychiatry; Human genome; Drug targets; Molecular pharmacology; Modern techniques; Neurotransmitters

\section{Introduction}

\subsection{Brain knowledge}

Suicide mechanisms in humans have not been fully understood worldwide. In the past, much progress of genotypic and phenotypic alteration and features for neurobiology of human suicide is associated with neurotransmitter, inorganic elements, organic chemical, ion transporter, synaptic connection, neural circuitry, cerebral image and facial movement [1-5]. The technical advances for human suicide and psychiatric disorders open a new era for understanding complex pathways, network and cerebral image of neurobiology in specific areas of human brain (volume, density and structure).

\subsection{Therapeutic dilemma for suicide}

The historic records of human suicide can be traced back as long as human activity took place. It is a basic human instinct behavior that is described by the ancient historians (Greece, Egypt and Rome for more than two thousand years) [5-6]. Unfortunately, no one took it serious at early historic records. Due to marginal activity of human suicide study over the past two thousand years, human suicide is not well manageable until now [1-4].

\section{Problem generation}

\subsection{Association with brain disorders}

As possible brain or mental disorders, an exploration for genetic alteration in patients with depression, bipolar, dysthymia, schizophrenia and others should be undertaken, especially those with suicide ideation and behaviors. Hidden rules and principles of suicide origin and pathological progress, especially aspects of diagnostic and therapeutic knowledge should be clarified. Unfortunately most mental diseases are chronic diseases that may accompany patients life-long (30 to 40 years or even longer), high-quality therapeutics are needed [6-10].

\footnotetext{
${ }^{*}$ Corresponding author: Dr. Da-Yong Lu

School of Life Sciences, Shanghai University, Shanghai200444, PRC.
} 
Although part of person's symptoms is mitigated from favorable clinical therapeutics with drugs or therapeutic combination, the rate of suicide ideations, events and mortality changes in a small-scale [11-15]. As a result, new perspective is needed. In order to overcome this limitation, some good clinical therapeutic paradigms should be established via new drug targets and categories [1-3].

\subsection{Novel mechanisms}

As unknown mechanisms for therapeutics against human suicide, sophisticated strategies and paradigms will be translated. To embrace modern scientific and technological advances, a number of unique diagnostic and therapeutic approaches should be sought after, such as wider-ranges of diagnostic information to different individuals (personalized medicine) and choice of higher effective drugs (appropriate drugs and dosages). Many unexpected therapeutic responses and outcomes (higher therapeutic-index and quality) may be realized after some novel drug development systems and clinical strategies.

\section{Pathology}

\subsection{Clinical limitation}

Patient's genetic variation or biomarkers (pharmacogenetics, PG and different omics profiling) are not widely used to guide therapeutics against human suicide behaviors [16-17]. Routine types of mental health diagnosis are not well balanced and high-efficiency. Also, many doctors do not know whole situation of patient's environmental or economic conditions due to limited times of patient-doctor interrogation and poor-statement by patient themselves. Facing this dilemma, psychiatric diagnostic and therapeutic modes should be replaced by biological entities drug targeting. The knowledge interplay and gaps at genetic, molecular, circuitry, visual and medicinal chemistry for suicide will be shown [18-19]. Only recently, its medical significance and controversy begin to be widely challenged.

\subsection{Drug safety}

Despite some favorable responses by antidepressants, especially Selective Serotonin Reuptake Inhibitors (SSRIs), controversy (no positive efficacies or even inductions of suicide events) can be seen in the past literatures [6-15]. By entering into this millennium, the US and European regulatory authorities began implementing verification programs to assess the influence on suicidal behavior from the use of antidepressants such as selective Serotonin Reuptake Inhibitors (SSRIs) [6-15]. A panic tension was rising among drug regulatory body since this millennium. Multidisciplinary factors or causalities for this therapeutic pandemic in the clinic will be the target of future. Genetic influence is proposed at this very beginning [20]. To mark this multi-disciplinary issue, the therapeutic variability among individual with suicide behaviors were carried out and compared with [11-13].

\subsection{Genes and neurotransmitters}

More than 400 human en-coding genes have been reported for association with human psychiatric behaviors and disorders [18-19]. Theoretically, at least 100-400 human encoding genes directly associated with human suicide. In the future, this association will be revealed one by one.

Multiple dimensions of mental illness origin and progress need different diagnostic or therapeutic means. Suicide ideations may undergo long-terms latency. Once a suicide/mental illness patient seeks psychiatric advises or suicide behaviors, the disease may have already been initiated for several years. The early therapeutic interventions may be the key for quick and thorough managements of suicide/mental illness symptoms. Prompt diagnostics of diseases by modern techniques may shorten the suicide identification and targeted treatments as early as possible. Certainly, new therapeutic models must be more helpful for suicide patients than ever before.

Table 1 Two major neurotransmitter systems [21]

\begin{tabular}{|c|c|c|c|}
\hline Neurotransmitters & Depleting agents & Level \& productions & Mental diseases \\
\hline \multirow{2}{*}{ Dopamine } & \multirow{2}{*}{$\begin{array}{l}\text { Branched-Chain Amino Acid } \\
\text { (BCAA) }\end{array}$} & Over-production & Schizophrenia \\
\hline & & Under-production & Neurodegenerative \\
\hline \multirow{2}{*}{ Serotonin } & \multirow{2}{*}{$\begin{array}{c}\text { 3,4-methylenedioxy } \\
\text { methamphetamine (MDMA) }\end{array}$} & Over-production & Anxiety/aversion \\
\hline & & Under-production & Depression \\
\hline
\end{tabular}




\subsection{Possible reasons for therapeutic difficulty}

- Long terms of disease latency

- Therapeutic choices are based on symptoms

- Different mental illness dimensions, such as different transmitter levels (Table 1)

- Large animal models are insufficiency

- Different genetic or molecular diagnosis is at early stage

\section{Major pathways}

\subsection{Technical frontiers}

As above-mentioned, the suicide/mental illness treatments need to be transformed from symptom evaluations (depression and mania) into molecular-guided diagnostics. Adapting new techniques into the clinical suicidal diagnostics is indispensable. Some of these new techniques have been utilized in multi-disciplinary and high-sensitivity.

\subsubsection{Technical solutions}

Some new techniques may help us to find out etiologic/pathologic causalities for individual patients-genes/epigenetic (genetic polymorphisms), neural molecules (transcription, enzymes, receptors or reuptake neurotransmitters) and anatomic-pathology (different types of neuron lesion or dysfunction) have been incorporated with potential clinical diagnosis [11].

\subsubsection{Classifications of disease dimensions}

Suicidal events and mortality are caused by different neuron damages (areas and density) and psychiatric symptoms (self-harm, self-injures and suicidal behaviors) (suicide tool take-away, different types of social or monetary supports) and neural-injury (neural death, $\beta$-amyloid $(A \beta)$ peptide aggregations, iron accumulation, neuro-inflammation and others)

\subsubsection{Pharmaceutical study (therapeutic drugs development and their clinical utility)}

Specific, highly effective, low costs, long-lasting and less toxic drug developments from the researches of medicinal chemistry, pharmacology and toxicology. Table 2 shows some of them.

Table 2 Neural biological pathways for suicide risks

\begin{tabular}{|l|l|l|}
\hline Neural & Targeted pathways & Effectors \\
\hline Ion channel & Na, K, Ca & Mg, Mn \\
\hline Transmitters & Monoamine & Serotonin, dopamine \\
\hline Synapse activity & Receptor, enzyme, reuptake & Neural function \\
\hline Signal receptors & 5-HT1A receptor & Neural impulsive \& functional \\
\hline Genes & MRP1, BCRP & Genotypic, SNP, copy number \\
\hline Molecule & Tau, $\beta$-amyloid peptide & Neural lesion and degeneration \\
\hline Cerebral & Amygdala, hippocampal, striatum & Aversion, anxiety and depression \\
\hline Neurons & Damage or degeneration & Eye ball or facial movement changes \\
\hline Psychiatric diseases & Cognitive, behavior or emotion & Schizophrenia, depression \\
\hline
\end{tabular}

\subsubsection{Clinical strategy and paradigms}

Clinical systems, such as gene-environment interaction, personalized (precision) medicine, genetics, omics and metabolomics and so on.

\subsection{Mental illness dimensions}

Generally speaking, a treatment of mental diseases is not easy. In many clinical occasions, these suicide or mental illness treatments are more complex than expecting for its long-term disease progress, a great variety of disease dimensions 
and variable therapeutic options. Due to the diverse dimensions of suicide causalities, the diagnostics and therapeutic systems should be personalized in disease diagnostics [22].

\subsection{Roads for future medication}

It is rarely seen that a high quality and straightforward diagnostics (genetic, bioinformatics and image systems) is available in general hospitals. New techniques and statistical analytical systems might be gradually established in the future. Yet, it has been recently found that environment impacts (bad experience and trauma in early days) can regulate and alternate gene expression, transcripts and epigenetics in sufferers since 2003 [18-19]. Many genes, molecules and receptors linking with dopaminergic and serotonergic systems can be regulated by different ranges of environmental forces and impacts. In early reports, epigenetic entities can be more dramatically changed in mental-illness patients than those of genetic variations [21].

\subsection{Algorithmic analysis and artificial intelligence (AI)}

State-of-the-art techniques and avant-garde instruments need data analysis, processing, identification, computation and statistics. Additionally, these state-of-the-art techniques need technicians to support and updating constantly. Mathematicians will handle algorithm, big-data, algebra and statistically analysis and finally theoretical generation for biological law and principle. It will be wonderful if some useful equation could be formed to support these mathematical issues.

\subsection{Clinical healthcare model for suicide}

Since suicide/mental disease pathogeneses are complex and multi-disciplinary, psychiatrists should be versatile. Yet, it is not so in real clinical settings. Some good therapeutic models are given for the interpretation of complex and mystery features of disease progresses.

A diagnostic and therapeutic tactics should be followed to enlarge therapeutic responses.

- Advanced knowledge of human mental illnesses, suicide risks and targeted therapeutics

- Show enough respects to suicide sufferers. As a result, patients will reveal their deep feelings and symptoms to doctors or clinicians

- Tactics in front of patients (avoid confrontations between doctors and patients and enlarge the number of patients willing to take medicine)

- Modest, willing to joint disease diagnosis with other types of experts or specialists if necessary (clinical teamwork)

- Learn information and knowledge from digital doctors so that the best technical assistance can be obtained

\subsection{From psychiatric genetics into suicidal genetics}

Psychiatric disorders are not $100 \%$ parallel with human suicide. The scientific approaches based on psychiatric disorders can be gradually translated into human suicide-leading to a vast space of imagination and promotion in both psychopathology and psychopharmacology. It is the key for therapeutic challenge and renovation for human suicide managements from neurobiological program and progresses.

\section{Future directions}

\subsection{Mechanism highlights and trends}

Suicide mechanism study will answer a lot of biomedical questions associated with human suicide behaviors and mortality. Following headlines are most important;

- Creative ideas can brood new generation of medical hypotheses and clinical therapeutics. This should be a priority for future advances

- New technique utilities such as Opto-genetics or Genetic Engineering Mice (GEM) experimental studies should be focused and support novel clinical diagnosis

- Drafting larger sample sizes of genetic, bioinformatics, pathophysiology evaluation between healthy and patients at high risks

- Genetic or genomic study of repeated DNA in human genomes for suicide diagnosis and drug discovery 
- The observation of interplay between genetics, molecular, chemical and a variety of environmental variables in suicide prediction, prevention and therapeutics

- Recruiting mathematics or physics major students and scholars in suicide studies

- Budget control of drug developments and clinical trials so that more patients can benefit from the therapy.

\subsection{Complex situation}

Clinical treatments for human suicide are not all dependent on drugs. The gene-gene and gene-environmental interaction are common pathways for neuroscience study. There are still many unanswered questions in the role of gene-driven or environmental driven mechanisms for the induction of suicide behaviors. The complex correlation of different disciplines is depicted in the figure. These different levels of suicide investigations are all integrated in futuristic trials and projects.

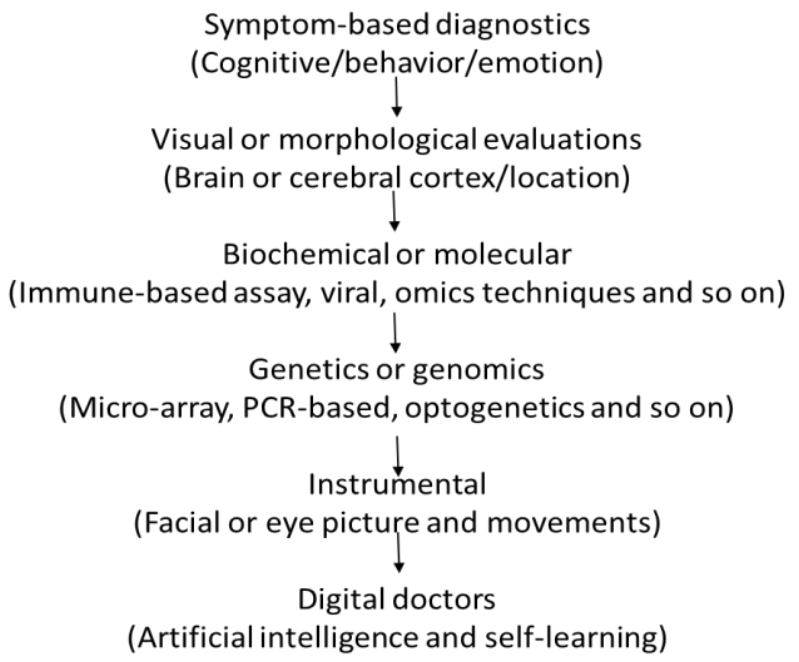

Figure 1 Technical evolutions for suicide prediction and prevention

\section{Conclusion}

In the future, expanding attentions should be paid off in the field of suicide/mental illness diagnosis and therapeutics [23-26]. Joint diagnosis and therapeutic decision-making by psychiatrists, pharmacologists, neurologists, biochemists, technicians and mathematicians can optimize suicide behaviors and therapeutic outcomes. We look forward to scientific and technical progress on personalize medicine.

\section{Compliance with ethical standards}

\section{Acknowledgments}

This article is supported by Shanghai Science and Technology Foundation of High Education 97A49.

\section{Disclosure of conflict of interest}

Bin $\mathrm{Xu}$ is also a retired professor of Shanghai Institute of Materia Medica, Chinese Academy of Sciences, PRC.

\section{References}

[1] Bondy B, Buettner A, Zill P. Genetics of suicide. Mol Psychiatry. 2006; 11: 336-351.

[2] Lu DY. Suicide Risks and Treatments, New Ideas and Future Perspectives. Ed Da-Yong Lu, Nova Science Publishers, New York, US. 2017.

[3] Lucas G. Fast-acting antidepressants: are we nearly there. Expert Rev Neurotherapeutics. 2008; 8(1): 1-3.

[4] Lu DY, Wu HY, Cao S, Che JY. Historical analysis of suicide. J Translational Genetics \& Genomics. 2020 ; 4: 33. 
[5] Shandilya S. Suicide and suicide prevention: a historical review. The Research Journal of Social Science. 2018; 9 (12): 35-40.

[6] Menchetti M, Bortolotti B, Rucci P, Scocco P, Bombi A, Berardi D. Depression in primary care: Interpersonal counseling vs selective serotonin reuptake inhibitors. The DEPICS study. A multicenter randomized controlled trial. Rationale and design. BMC Psychiatry. 2010; 10: 97.

[7] Menchetti M, Rucci P, Bortolotti B, Bombi A, Scocco P, Kraemer HC, Berardi D. Moderators of remission with interpersonal counseling or drug treatment in primary care patients with depression: randomized controlled trial. Br J Psychiatry. 2014; 204(2): 144-150.

[8] Serafini G, Salano P, Amore M. Suicidal ideation: a comprehensive overview. Suicidal Ideation: Predictors, Prevalence and Prevention. Ed. Bradley Weaver. Nova Science Publishing. US. 2015; 1: 1-42.

[9] Rubino A, Roskell N, Tennis P, Mines D, Weich S, Andrews E. Risk of suicide during treatment with venlafaxine, citalopram, fluoxetine, and dothiepin: retrospective cohort study. BMJ. 2007; 334(7587): 242-247.

[10] Kovacs D, Gonda X, Petschner P, Edes A, Eszlari N, Bagdy G, Juhasz G. Antidepressant treatment response is modulated by genetic and environmental factors and their interactions. Annals General Psychiatry. 2014; 13(1): 17.

[11] Lu DY, Lu TR, Che JY, Zhu PP. Genetics and bioinformatics studies of antidepressant drug therapeutic efficacies and toxicities, a current overview. Recent Pat CNS Drug Discov. 2014; 9(3): 193-199.

[12] Lu DY, Lu TR, Zhu PP, Che JY. The efficacies and toxicities of antidepressant drugs in clinics, building the relationship between Chemo-Genetics and Socio-Environments. Cent Nerv Syst Agents Med Chem. 2016; 16(1): 12-18.

[13] Lu DY, Lu TR, Zhu PP, Che JY. Genetics and bioinformatics study of antidepressant drugs, recent advancements and future trends. Suicidal Ideation: Predictors, Prevalence and Prevention. Ed. Bradley Weaver. Nova Science Publishing. US. 2015; 3: 57-71.

[14] Lu DY, Zhu PP, Lu TR, Che JY. The suicide risks and treatments, seek medications from multi-disciplinary. Cent Nerv Syst Agents Med Chem. 2016; 231-239.

[15] Maher B. Genomes on prescriptions. Nature. 2011; 478(7367): 22-24.

[16] Kuo HW, Liu C, Tsou HH, Liu SW, Lin M, Lu SC, Hsiao MF, Hsiao CF, Liu CY, Chen CH, Lu ML, Shen WW, Tang HS, Liu SI, Chang LH, Wu HY, Chang YS, Yeh TK, Chen AC, Liu YL. CYP1A2 genetic polymorphisms are associated with early antidepressant escitalopram metabolism and adverse reactions. Pharmacogenomics. 2013; 14(10): 11911201.

[17] Lu DY, Lu TR, Zhu PP. Pharmacogenetics in neural toxicities of drugs. Pharmacogenomics. 2013; 14(10): 11291131.

[18] Malhotra D, Sebat J. CNVs: harbingers of a rare variant revolution in psychiatric genetics. Cell. 2012; 148: 12231241.

[19] Krystal JH, State MW. Psychiatric disorders: diagnosis to therapy. Cell. 2014; 157: 201-214.

[20] Lu DY, Lu TR, Ding J. May genetic factors play a role in the risk of antidepressant-induced suicide. Med Hypotheses. 2007; 69(6): 1380-1381.

[21] Ramsay TZ, Skov M. How genes make up your mind: Individual biological differences and value-based decisions. J Economic Psychology. 2010; 31: 818-831.

[22] Marshall M. Roots of mental illness. Nature. 2020; 581: 19-21.

[23] Read J, Runciman 0, Dillion J. In search of an evidence-based role for psychiatry. FSOA. 2016; 2: 1.

[24] Reardon S. Brain study seeks roots of suicide. Nature. 2015; 528: 7580.

[25] Lu DY, Cao S, Xu B, Lu Y, Lu TR. Suicide prevention and treatments. Novel Approach Drug Design \& Develop. 2017; 1(4): 555-582.

[26] Lu DY, Wu HY, Lu TR. Human suicide study, new insights and drug development. J Community Medicine. 2020; 3 (1): 1028. 